\title{
Modified Smallpox
}

National Cancer Institute

\section{Source}

National Cancer Institute. Modified Smallpox. NCI Thesaurus. Code C35028.

Smallpox that occurs in an individual who has been vaccinated. 